priming of antitumour $\mathrm{CD} 8^{+} \mathrm{T}$ cells in the DCLNs of control animals with glioblastoma; by contrast, VEGF-C treatment markedly enhanced the priming of $\mathrm{CD}^{+} \mathrm{T}$ cells against the brain tumours.

Finally, the authors found that co-treatment with VEGF-C and anti-PD1 also promoted antitumour immunity against brain tumours derived from melanoma cell lines. Notably, VEGF-C did not potentiate the efficacy of anti-PD1 therapy if the mice had both intracranial and flank melanoma-derived tumours.

These findings support the idea that $\mathrm{T}$ cell priming is necessary for the efficacy of immune checkpoint inhibitors in brain tumours. Therefore, factors such as VEGF-C that increase lymphatic drainage and enhance $\mathrm{T}$ cell priming may increase the efficacy of immune checkpoint blockade for tumours affecting the brain and other immune-privileged sites.

Yvonne Bordon

ORIGINAL ARTICLE Song, E. et al. VEGF-C-driven lymphatic drainage enables immunosurveillance of brain tumours. Nature https://doi.org/10.1038/ 541586-019-1912-x (2020)

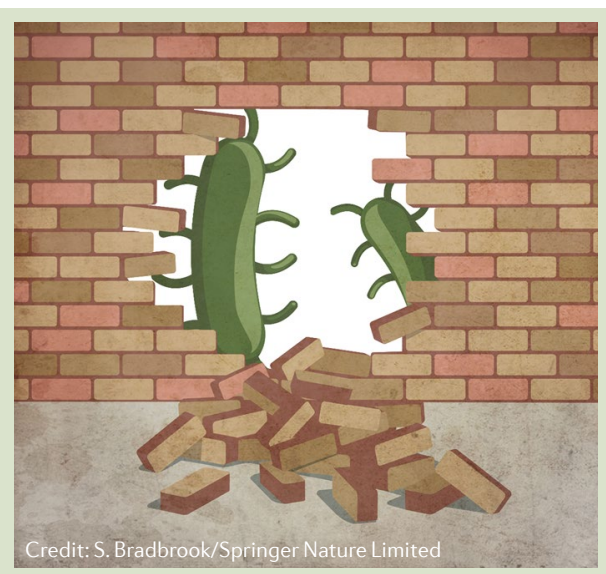

Thus, differentiated root cells appear to require a double hit of local cellular damage and MAMP exposure to upregulate MAMP-responsive genes. Such damage-gating may enable the colonization of roots by innocuous or beneficial bacteria and restrict potentially detrimental immune responses to aggressive microbial colonizers.

Yvonne Bordon

ORIGINAL ARTICLE Zhou, F. et al. Co-incidence of damage and microbial patterns controls localized immune responses in roots. Cell 180 , 440-453 (2020)

\title{
MR1-restricted
}

\section{pan-cancer T cells}

Current T cell-based cancer therapies are personalized to each patient (to ensure HLA matching) and are only suitable against certain cancers (with known target antigens). So the idea of a T cell-based therapy that is not limited by polymorphic MHC restriction or known tumour antigens is very attractive. A study published in Nature Immunology describes a new $T$ cell population that specifically kills many human cancer cell types via recognition of the non-polymorphic MHC class I-related protein MR1. MR1 is widely expressed and varies little between individuals, raising the prospect of a universal cancer immunotherapy.

A T cell population that proliferated in response to A549 cancer cells was isolated from peripheral blood mononuclear cells from an HLA-mismatched healthy donor. Remarkably, the resultant $\mathrm{T}$ cell clone named MC.7.G5 - could kill multiple cancer cell lines regardless of their HLA allomorph expression and did not kill healthy cells. The authors used a genomewide CRISPR-Cas9 screening approach to identify the genes essential for recognition of target cells by MC.7.G5 T cells. In this screen, only transduced target cells in which the key gene involved in recognition was ablated survived selection with MC.7.G5 $T$ cells. This led to the identification of six genes in the resistant target cells: B2M, MR1, STAT6 and three genes associated with transactivation of $\beta_{2} \mathrm{M}$ and $\mathrm{MHC}$ class I and II promoters (RFX, RFXANK and RFXAP), suggesting that MC.7.G5 $\mathrm{T}$ cells recognize cancer targets via MR1, which forms heterodimers with $\beta_{2} \mathrm{M}$. Accordingly, anti-MR1 antibody, but not MHC class I or II antibodies, blocked target cell recognition by MC.7.G5 T cells. In addition, reintroduction of MR1 into CRISPR-Cas9mediated MR1-knockout A549 cells restored recognition by MC.7.G5 T cells.

MR1 molecules presenting intermediates of

riboflavin synthesis are known to be sensed by

mucosal-associated invariant T (MAIT) cells. But MC.7.G5 T cells did not recognize MR1 bound with MAIT cell-activating ligands nor empty MR1 molecules or MR1 expressed by healthy monocyte-derived dendritic cells. Moreover, target cell recognition by MC.7.G5 T cells was reduced in the presence of riboflavin-derived microbial MR1 ligands. This suggested that MC.7.G5 T cells recognize MR1 molecules presenting cargo that is specific to cancer cells. Importantly with respect to their potential use as cancer immunotherapy, MC.7.G5 $T$ cells remained unresponsive to healthy cells from various tissues even after MR1 surface expression was upregulated by cell activation or after exposure to cell stressors.

To test their capacity to target cancer in vivo, MC.7.G5 T cells were adoptively transferred into mice engrafted with leukaemia cells. Mice receiving MC.7.G5 T cells showed a marked reduction in cancer burden 18 days later and increased survival compared with mice that did not receive MC.7.G5 T cells. This in vivo targeting was dependent on expression of MR1 by the leukaemia cells, as shown by co-transfer of wild-type and $M R 1^{-/-}$leukaemia cells. Finally,

\section{(40-453 (2020)}

therapeutic potential was highlighted by the finding that $\mathrm{T}$ cells purified from patients with stage IV melanoma and transduced with the MC.7.G5 T cell receptor could recognize and kill autologous and non-autologous melanomas but not healthy cells or $M R 1^{-/-}$melanomas.

This paper identifies an intriguing new population of cancer-specific $T$ cells that has potential as a pan-cancer therapy. Future work will be needed to establish the exact nature of the ligand recognized by the MC.7.G5 T cells.

Lucy Bird

ORIGINAL ARTICLE Crowther, M. D. et al. Genome-wide CRISPRCas9 screening reveals ubiquitous $T$ cell cancer targeting via the monomorphic MHC class I-related protein MR1. Nat. Immunol. 21, 178-185 (2020) 